\title{
The Representational Nature of Representational Noun Phrases
}

\section{Micah B. Goldwater, University of Texas at Austin \& Jeffrey T. Runner, University of Rochester}

\section{Introduction}

Question

Why are reflexives in representational noun phrases (e.g. a picture of himself) exempt from Binding Theory?

\section{Two hypotheses:}

- Linguistic Structure: reflexives in certain lexical argument structures are exempt from Binding Theory (Pollard \& Sag 1992, Reinhart \& Reuland 1993); or representational Noun Phrases (RNP's) contain a null possessor which binds the reflexive (e.g. Chomsky 1986, Davies \& Dubinsky 2003).

- Representational Meaning: RNP reflexives are different from "normal" reflexives because they refer to representations of people, not because of linguistic structure.

\section{Background}

Parallels between "Reference Transfer" reflexives and RNP reflexives

- Jackendoff (1992) notes that "reference transfer"(RT) allows a representation of a person to be referred to by a reflexive, but only true referent can bind reflexive.

1. At the wax museum of music history, Ringo Starr saw himself.

2. Ringo fell on himself. <only if real Ringo falls on statue $>$

- Lidz (2001) points out that these reflexives (unlike "normals") can co-refer when elided. 3. Ringo Starr saw himself, and Paul did too. $<$ saw statue of Ringo>

- RNP reflexives also seem to be able to co-refer when elided.

4. Bill admired the picture of himself, and Beth did too. $<$ admired picture of Bill $>$

- RT and RNP reflexives each refer to representations of people but appear in different linguistic structures; do they behave similarly for the same reason?

\section{Experiment 1}

\section{Question}

Do RNP reflexives receive more co-referential interpretations than "normal" direct object reflexives?

\section{Methods}

- 23 participants saw scenes on a monitor and heard prerecorded sentences.

- verified if the sentence matched the scene by pressing ' $y$ ' or ' $n$ '.

- Each sentence contained one of three verbs.

- 12 critical sentences with ellipsis, 12 control with no ellipsis, and 48 fillers with no reflexives.

- 2 critical sentence types, each matched with 2 different scenes (see Display $1 \& 2$, column 2)

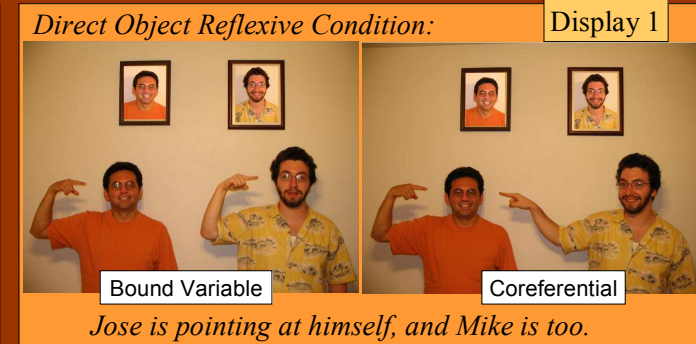

RNP Reflexive Condition:

Display 2
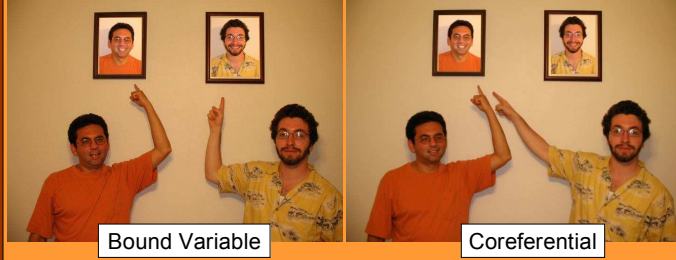

Jose is pointing at a picture of himself, and Mike is too.

\section{Results \\ Fig. 1. Proportion of Coreferential \\ Responses Exp 1.}

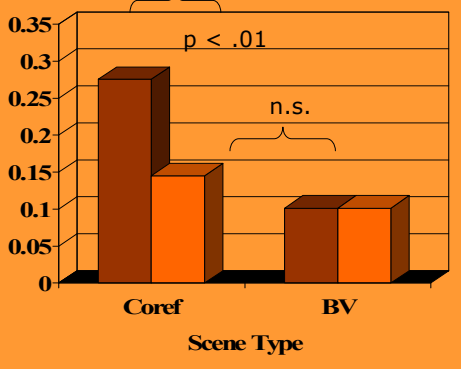

$\square \mathbf{R N}$ $\square$ OBJ

a coreferential response is a " $\mathrm{n}$ "

There was a marginal interaction of scene type $\mathrm{x}$ reflexive type $p<$ 1. Importantly, the planned comparison of RNP vs OBJ within the coref scene was significant, $p<.01$ (one-tailed)

-Summary: Elided RNP reflexives more likely to receive coreferential interpretations than OBJ reflexives.

\section{Experiment 2}

\section{Question}

-Will reference transfer reflexives also co-refer more than normal reflexives? Exp 1 had correlated linguistic structure and referent of the reflexive. Exp 2 decouples the two to isolate the effect of structure.

\section{Methods}

- Same scenes as Experiment 1

- all reflexives appear as direct objects - no RNP's.

- Half of reflexives are RT reflexives, half "normal" - $\mathrm{N}=37$

Normal Reflexive Condition: see Display 1, above.

RT Reflexive Condition:

Display 3
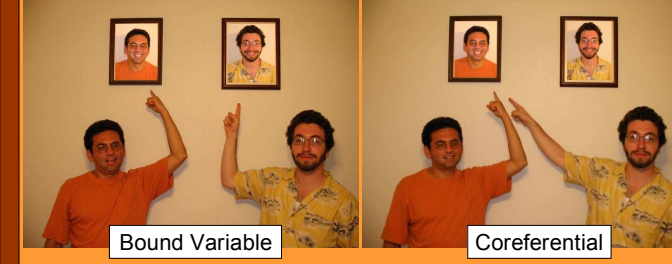

Jose is pointing at himself, and Mike is too.

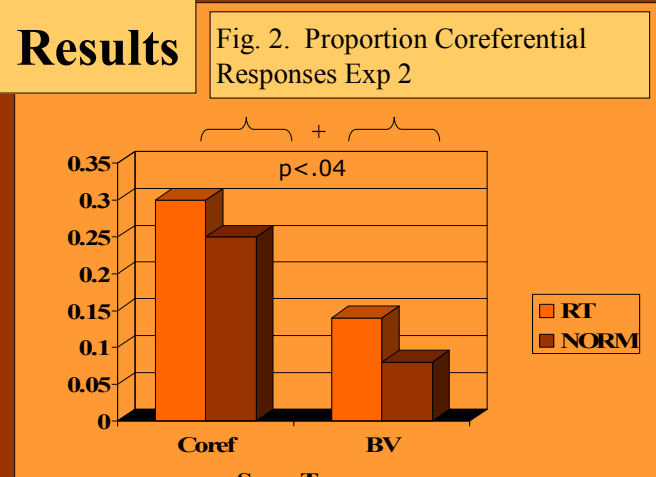

Note: The No-ellipsis BV scene allowed us to see if any participant would not accept reference transfer. If a participant rejected at least 2 out of the 3 trial of this type they were excluded from analysis; 6 out of 37 were excluded.

There was a main effect of reflexive type (i.e. to what the reflexive referred), $p<.04$

- Summary: Elided RT reflexives more likely to receive coreferential interpretations than normal OBJ reflexives.

Comparing Exp 1 \& 2: RNP vs. RT

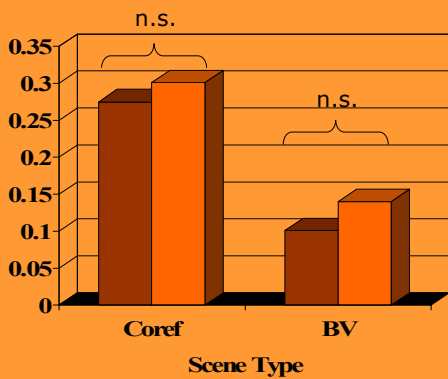

Fig. 3

There were no effects of NP structure, all $f$ 's $<.025$.

Summary: Reflexives behaved the same when they referred to a representation regardless of linguistic structure.

\section{Conclusions \& Future Directions}

- Results inconsistent with either linguistic structural account.

- The referential distinction was only in the visual scene (Exp 2) so could have only been instantiated in a "non-linguistic conceptual" representation.

- The nature of this instantiation will be the subject of future research.

- Eye-tracking version of these studies is currently being run to investigate the implicit processes leading to these interpretations.

\section{References}

Chomsky, N (1986) Knouledge of Language. New York; Praeger Davies \& Dubinsky (2003). On extraction from NP's. Natural Language \& Linguistic Theor? . Tussaud meets the binding theory. Natural Language \& Linguistic Lidz, J. (2001). Condition R. Linguistic Inquiry, 32, 123-140. Reinhart, T. \& Reuland, E. (1993). Reflexivity. Linguistic Inquiry, 24, 657-720. Pollard, C. \& Sag, L. (1992). Anaphors in English and the scope of Binding Theory. Linguistic Inquiry, 23, 261-303.

Micah Goldwater: micahbg@mail.utexas.ed 